\title{
A Tunable Encoder/Decoder Based on Polarization Modulation for the SAC-OCDMA PON
}

\author{
He Chen, Shilin Xiao, Lilin Yi, Zhao Zhou, Min Zhu, Jie Shi, Yi Dong, Member, IEEE, and \\ Weisheng $\mathrm{Hu}$, Member, IEEE
}

\begin{abstract}
We propose a tunable encoder/decoder based on polarization modulation for the spectrum amplitude coded-optical code-division multiple access (SAC-OCDMA) passive optical network (PON) and experimentally demonstrate its coding/decoding performance for the first time. The experiment result verifies that the encoder/decoder with 40-dB blocking ratio can fully suppress the unwanted spectral slices and performs good coding/decoding capability. Code length of 45 and code weight of 9 are demonstrated for proof-of-concept.
\end{abstract}

Index Terms-Fiber optical communications, liquid crystals, multiplexing.

\section{INTRODUCTION}

$\mathbf{S}$ PECTRAL amplitude coded-optical code division multiple access (SAC-OCDMA) is one of promising approaches for optical access network by taking advantages of asynchronous transmission, tell and go access protocol, soft capacity on demanding and huge degree of scalability [1]-[3]. A series of components have been used for spectrum amplitude coding, such as acoustically tunable optical filter (ATOF) [4]-[6], Fabry-Pérot filter [7], and fiber Bragg gratings (FBGs) [8]-[12]. Their coding capability has been verified by experiment. However, the Fabry-Pérot filter and FBG based coding scheme are difficult to reconfigurable, and the ATOF needs radio-frequency driving signal. Then the opto-very large integrated (Opto-VLSI) processor is exploited to be the encoder/decoder for the SAC-OCDMA system and provides reasonable coding performance [13].

In this letter, we propose a tunable encoder/decoder based on polarization modulation for the SAC-OCDMA PON and experimentally demonstrate its coding/decoding performance for the first time. The encoder/decoder is constructed with liquid crystal material, and it is reconfigurable and with high blocking

Manuscript received January 07, 2011; revised February 19, 2011; accepted March 16, 2011. Date of publication March 24, 2011; date of current version May 13, 2011. This work was supported by the National Nature Science Fund of China (60972032 and 61007041), and by the National "863" Hi-tech Project of China (2006AA01Z251 and 2007AA01Z271).

H. Chen is with State Key Laboratory of Advanced Optical Communication Systems and Networks, Department of Electronic Engineering, Shanghai Jiao Tong University, Shanghai 200240, China, and also with the School of Information Science and Engineering, Xinjiang University, Urumqi 830046, China (e-mail: gravelhe@gmail.com).

S. Xiao, L. Yi, Z. Zhou, M. Zhu, J. Shi, Y. Dong, and W. Hu are with the State Key Laboratory of Advanced Optical Communication Systems and Networks, Department of Electronic Engineering, Shanghai Jiao Tong University, Shanghai 200240, China (e-mail: slxiao@sjtu.edu.cn; lilinyi@sjtu.edu.cn; yidong@sjtu.edu.cn; wshu@sjtu.edu.cn).

Color versions of one or more of the figures in this letter are available online at http://ieeexplore.ieee.org.

Digital Object Identifier 10.1109/LPT.2011.2132701

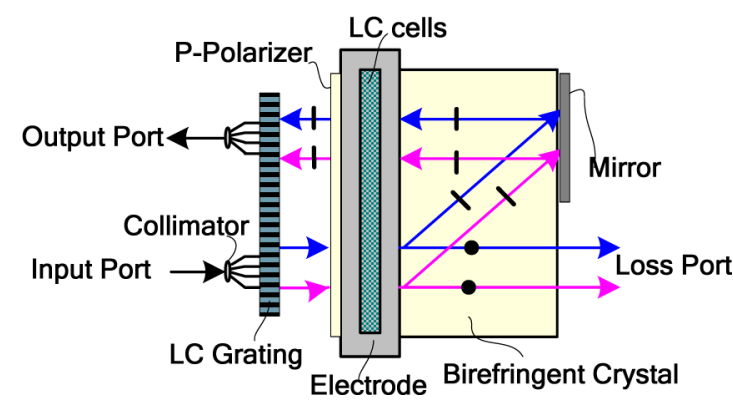

Fig. 1. Optical path of encoder/decoder.

ratio of about $40 \mathrm{~dB}$ for unwanted spectral slices, whereas all the previous encoders only have about $20-\mathrm{dB}$ blocking ratio (ATOF: $20 \mathrm{~dB}$; FBGs: $20 \mathrm{~dB}$; Opto-VLSI's: $25 \mathrm{~dB}$ ).

\section{ENCODER/DECODER BASEd ON POLARIZATION MODULATION}

Liquid crystal (LC) material is frequently used in wavelength selective switches (WSSs). When a voltage is applied across an LC material, the molecular orientation of the $\mathrm{LC}$ becomes aligned in a known direction, therefore changes the polarization state of incident light. For twisted-nematic (TN) LC material and initially p-polarized light, $99.99 \%$ light will still be p-polarized when the voltage is larger than $4 \mathrm{~V}$, and $99.9 \%$ light will be s-polarized under zero voltage [14]. Therefore the spectral component of the output port can be tuned flexibly by the electrode voltage, which can be used for spectral amplitude coding in OCDMA system.

Here we design an encoder/decoder based on the polarization modulation of the TN LC. As shown in Fig. 1, when the broadband optical lights from the input port are transmitted into the LC grating through a collimator, different wavelengths are separated in space (such as red line and blue line in Fig. 1) and then are incident on different LC cells. Every cell conditioned independently by the electrode will modulate the polarization state of the individual wavelength, which will be directed to the output port or the loss port base on the voltage applied on each cell. Therefore the spectral component of the output port can be tuned flexibly by the electrode voltage, which can be used for spectral amplitude coding in OCDMA system.

The high blocking ratio of the proposed encoder/decoder can be explained as below. As shown in Fig. 1, the lights separated in space by the LC grating are p-polarized after a p-polarizer. We define the wavelengths to the output port as on-wavelength and the ones to the loss port as off-wavelength. When we tune the electrode voltage of the LC cells corresponding to the on-wavelength to $4 \mathrm{~V}$, the polarization state of the on-wavelength will not change and still be p-polarized (vertical bar "|" shown in Fig. 1). 


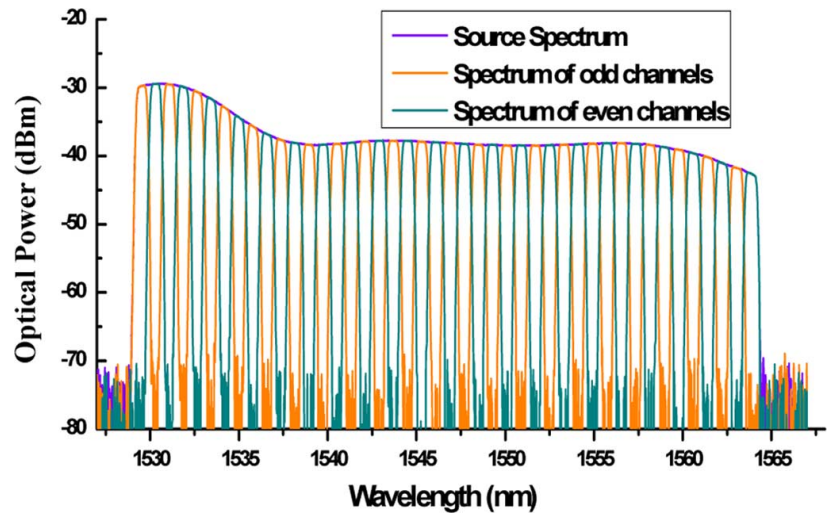

Fig. 2. Block ratio of the $\mathrm{LC}$ encoder/decoder.

Then it is guided to the up-path and directed to the output port through a mirror and the LC cells, and only $0.01 \%$ light is lost and leaked to the loss port. For achieving the off-wavelength at the loss port, we can tune its corresponding LC cells' voltage to zero. Then $99.9 \%$ light becomes s-polarized (solid dot "•" shown in Fig. 1) and is directed to the loss port, while the other $0.1 \%$ light becomes p-polarized and is guided to the up-path. For the up-path p-polarized light, only $0.1 \%$ light is still p-polarized after the LC cells at 0 driving-voltage and is directed to the output port, while the other $99.9 \%$ light becomes s-polarized and is blocked by the p-polarizer. Therefore only $0.01 \%$ light of the off-wavelength is leaked to the output port, corresponding to $40-\mathrm{dB}$ blocking ratio. Fig. 2 shows the blocking performance of the proposed encoder/decoder. The unwanted spectral is blocked to lower than $-70 \mathrm{dBm}$ from the original optical source power of about $-30 \mathrm{dBm}$.

A tunable encoder/decoder with high blocking ratio for SACOCDMA can be exploited based on the above principle. The coding/decoding scheme is achieved by controlling the voltage of the corresponding LC cells to be $4 \mathrm{~V}$ or $0 \mathrm{~V}$ based on " 1 " or " 0 " code in code chips respectively.

\section{Merit of High On/OfF Ratio}

Non-fully blocking of the unwanted spectrum slices in encoder/decoder is also an inherent noise in the SAC-OCDMA system. The intensity is assumed to be 1 if the spectrum slice is on, and zero if the spectrum is ideally off. But the nonfully blocking of the unwanted spectrum slices will lead the intensity to be $e^{-E R / 10}$ when the spectrum is off, where ER expresses the blocking ratio of the encoder/decoder. Just regarding of the inherent noise of encoder/decoder and multiple users' interference (MUI), the signal to noise ratio (SNR) is deduced to be:

$$
\begin{aligned}
\mathrm{SNR} & =\frac{\omega \times 1+(N-\omega) \times e^{-\mathrm{ER} / 10}}{(K-1) \times \lambda+(K-1) \times(N-\lambda) \times e^{-\mathrm{ER} / 10}} \\
& =\frac{\omega \times 1+(N-\omega) \times e^{-\mathrm{ER} / 10}}{(K-1) \times\left(\lambda+\times(N-\lambda) \times e^{-\mathrm{ER} / 10}\right)}
\end{aligned}
$$

where $\mathrm{N}$ is the code length, $\omega$ is the code weight, $\lambda$ is the crosscorrelation of codeword, and $K$ is the number of active users employed in the SAC-OCDMA system. From the above equation, it is worthy to note that $(K-1) \times(N-\lambda) \times e^{-\mathrm{ER} / 10}$ can't be neglected comparing with multiple users interference of $(K-1) \lambda$ when the blocking ratio ER is about $20 \mathrm{~dB}$. But if the ER is $40 \mathrm{~dB}$, the inherent noise in the encoder/decoder

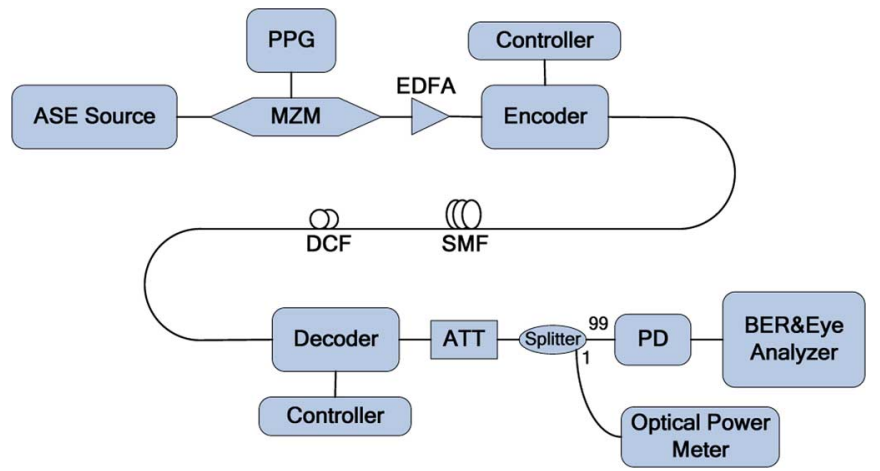

Fig. 3. Experimental setup for tunable encoder/decoder.

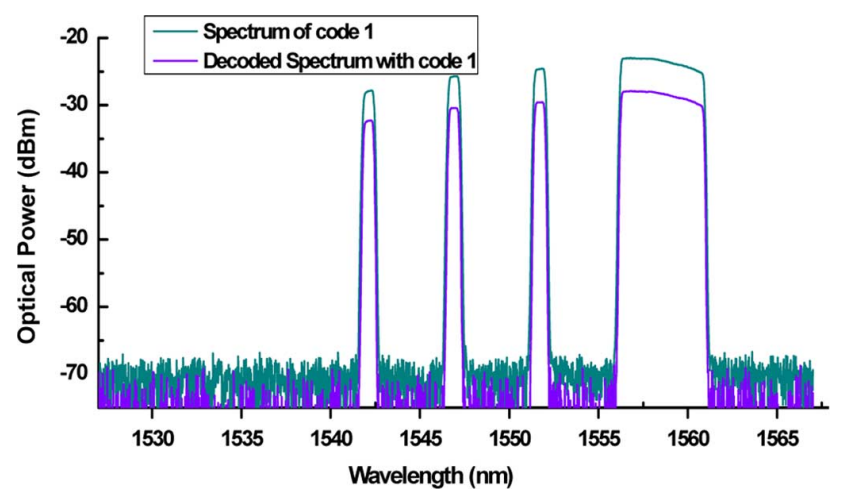

Fig. 4. Spectral slice of Code 1 .

can be ignored. So the encoder/decoder exploited in this letter is beneficial to interference suppression.

\section{EXPERIMENT OF THE TUNABLE ENCODER/DeCODER FOR THE SAC-OCDMA PON}

In experiment demonstration, we choose enhanced double weight (EDW) [15] as the code. The code length is 45 , and the code weight is 9 . The spectral slices are spaced by $0.8 \mathrm{~nm}$ from $1528.77 \mathrm{~nm}$ to $1564.68 \mathrm{~nm}$. The experiment setup schematic is shown in Fig. 3.

In the experiment, the amplified spontaneous emission (ASE) noise from an erbium doped fiber amplifier (EDFA) is used as a broadband source in $C$-band, and it is modulated by a Mach-Zehnder modulator (MZM) with a $2.5-\mathrm{Gb} / \mathrm{s}$ nonreturn-to-zero (NRZ) PRBS data $\left(2^{31}-1\right)$ from a pulse pattern generator (PPG). After being amplified by another EDFA, the signal is coded by the encoder. At the receiver side, the signal is decoded and then detected by a photodiode (PD). Controllers are employed to tune the code used in the encoder and decoder. For compensating the strong dispersion of the broadband source, 170-ps/nm dispersion compensation fiber (DCF) is employed after the 12.9-km single mode fiber (SMF). An attenuator (ATT) is used to vary the received optical power during the measurement of bit error rate (BER). An optical splitter is assigned after the ATT for dividing $99 \%$ received power to the PD and the other $1 \%$ power to the optical power meter.

Firstly, we tune the code address of both encoder and the decoder to code 1: 00000111111000001000001000001000000 0000000000 by controllers. Fig. 4 shows the coded spectral component by code 1 and the decoded spectrum when the decoder matches with the encoder. The high blocking ratio of the 


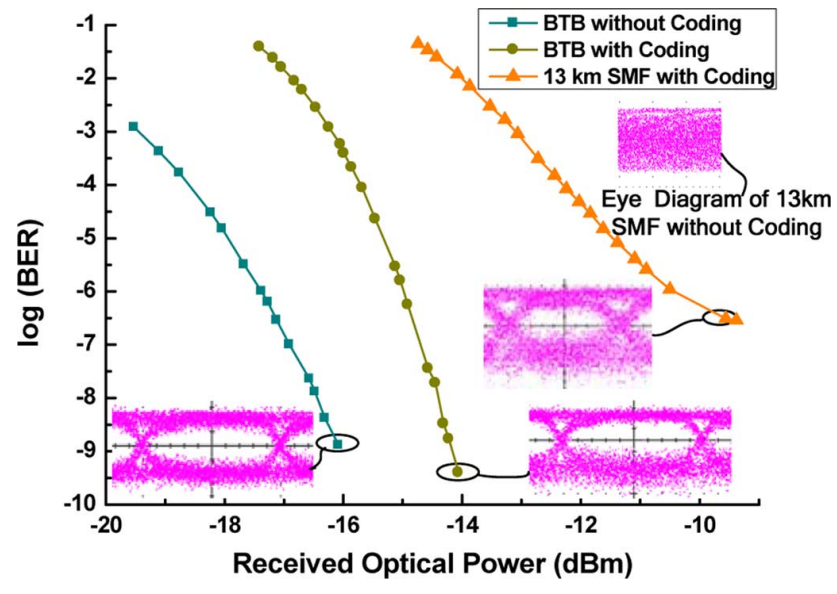

Fig. 5. BER and eye diagram measurement.

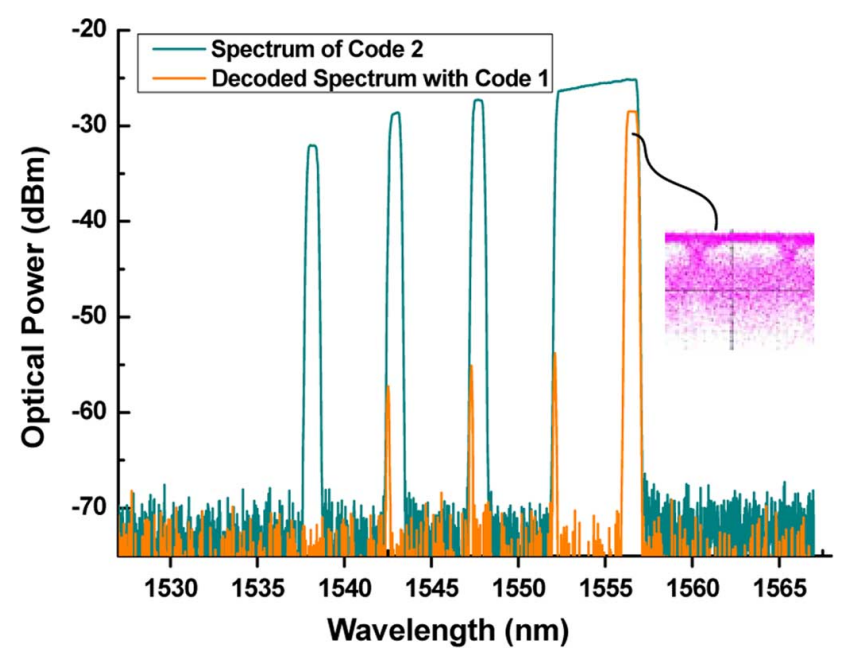

Fig. 6. Spectral slice and eye diagram of unmatched decoding.

unwanted spectral slices is a guarantee of large number users. The total loss of the proposed encoder/decoder is about $7 \mathrm{~dB}$, and every encoder or decoder may introduce $3.5 \mathrm{~dB}$ insertion loss. Then the coding performance is measured as shown in Fig. 5. The BERs and eye diagrams of back to back (BTB) transmission with coding are a little degraded compared with those without coding, since part of the optical spectrum is lost after coding and the encoder and the decoder may not be perfect matched. But when the transmission range is $13 \mathrm{~km}$ and no coding is employed, the dispersion noise is dominant and the eye closes completely even at $0-\mathrm{dBm}$ received power as shown in Fig. 5. When the code used in encoder and the decoder is matched, the eye becomes open again since the dispersion is reduced after spectral amplitude coding.

The encoder/decoder is designed for the SAC-OCDMA system, so the eye diagram of unmatched code addresses is supposed to be closed. Here, we tune code address of the encoder to code 2: 000000000011111100000100000100 000100000000000 , but maintain the decoder's code address be code 1 . The cross-correlation between code 1 and code 2 will exist in the decoded spectrum (orange part in Fig. 6), and it is the interference noise, so the eye diagram of BTB transmission is not fully closed. It is clear that there are another 3 spectrum slices (about $-60 \mathrm{dBm}$ ) appearing in the decoded spectrum.
They are the inherent interference from the adjacent channels in the encoder, but they are much less compared with the original spectrum, therefore can be ignored. After $13 \mathrm{~km}$ fiber transmission, the eye diagram becomes much worse, so the signal can not be recovered from the unmatched encoder/decoders, which proves our proposed encoder/decoder can be used in SAC-OCDMA PON systems.

\section{CONCLUSION}

We have proposed a tunable encoder/decoder based on polarization modulation of LC materials for the SAC-OCDMA PON. The encoder/decoder not only has the merit of flexible reconfiguration with code addresses but also has high blocking ratio for the unwanted spectral slices. The experiment verifies that the blocking ratio is more than $40 \mathrm{~dB}$, whereas the previous coding devices only have 20 -dB blocking ratio, so it may be a promising option for the encoder/decoder in the SAC-OCDMA PON.

\section{REFERENCES}

[1] D. Zaccarin and M. Kavehrad, "An optical CDMA system based on spectral encoding of LED," IEEE Photon. Technol. Lett., vol. 5, no. 4, pp. 479-482, Apr. 1993.

[2] M. Kavehrad and D. Zaccarin, "Optical code-division-multiplexed systems based on spectral encoding of noncoherent sources," J. Lightw. Technol., vol. 13, no. 3, pp. 534-545, Mar. 1995.

[3] L. Adam, E. S. Simova, and M. Kavehrad, "Experimental optical CDMA system based on spectral amplitude encoding of noncoherent broadband sources," in All-Optical Communication Systems: Architecture, Control, and Network Issues. Philadelphia, PA: SPIE, 1995, pp. 122-132.

[4] K. Iversen and O. Ziemann, "An all-optical CDMA communication network by spectral encoding of LED using acoustically tunable optical filters," in Proc. URSI Int. Symp. Signals, Systems, and Electronics, 1995, pp. 529-532.

[5] I. Hinkov et al., "Feasibility of optical CDMA using spectral encoding by acoustically tunable optical filters," Electron. Lett., vol. 31, pp. 384-386, 1995.

[6] O. Ziemann and K. Iversen, "Optical CDMA Based on Spectral Encoding With Integrated Optical Devices," in All-Optical Communication Systems: Architecture, Control, and Network Issues. Philadelphia, PA: SPIE, 1995, pp. 142-152.

[7] T. Pfeiffer et al., "High speed optical network for asynchronous multiuser access applying periodic spectral coding of broadband sources," Electron. Lett., vol. 33, pp. 2141-2142, 1997.

[8] G. A. Magel et al., "Transmission of eight channels $\times 622 \mathrm{Mbit} / \mathrm{s}$ and 15 channels $\times 155 \mathrm{Mbit} / \mathrm{s}$ using spectral encoded optical CDMA," Electron. Lett., vol. 37, pp. 1307-1308, 2001.

[9] M. Julien et al., "Experimental demonstration of frequency-encoded optical CDMA using superimposed fiber Bragg gratings," in Bragg Gratings, Photosensitivity, and Poling in Glass Waveguides, 2003, p. WD4, OSA/BGPP.

[10] S. Ayotte et al., "Experimental verification and capacity prediction of FE-OCDMA using superimposed FBG," J. Lightw. Technol., vol. 23, no. 2, pp. 724-731, Feb. 2005.

[11] J. Penon et al., "Spectral-amplitude-coded OCDMA optimized for a realistic FBG frequency response," J. Lightw. Technol., vol. 25, no. 5, pp. 1256-1263, May 2007.

[12] J. Penon et al., "An innovative receiver for incoherent SAC-OCDMA enabling SOA-Based noise cleaning: Experimental validation," $J$. Lightw. Technol., vol. 27, no. 2, pp. 108-116, Jan. 15, 2009.

[13] M. Aljada, S. Hwang, and K. Alameh, "Design of 10 Gbps optical encoder/decoder structure for FE-OCDMA system using SOA and optoVLSI processors," Opt. Express, vol. 16, pp. 679-685, 2008.

[14] H. P. Yuan, W. Xuehua, L. Christopher, and B. Giovanni, "High Extinction Ratio Liquid Crystal Optical Switch," U.S. Patent, 2008, Union City, El Cerrito, Saratoga, CA, CA, CA, CA,

[15] A. Z. G. Zahid, F. N. Hasoon, and S. Shaari, "New code structure for enhanced double weight (EDW) code for spectral amplitude coding OCDMA system," in Proc. Int. Conf. Future Computer and Communication, 2009, pp. 658-661. 